\title{
Outcome in Patients with High Body Mass Index following Primary Total Hip Arthroplasty
}

\author{
Zuned Hakim, Claire Rutherford, Elizabeth Mckiernan, and Tony Helm \\ Royal Preston Hospital, Sharoe Green Lane North, Fulwood, Preston PR2 9HT, UK \\ Correspondence should be addressed to Zuned Hakim; zuned.hakim@gmail.com
}

Received 16 December 2014; Accepted 29 April 2015

Academic Editor: Werner Kolb

Copyright (C) 2015 Zuned Hakim et al. This is an open access article distributed under the Creative Commons Attribution License, which permits unrestricted use, distribution, and reproduction in any medium, provided the original work is properly cited.

\begin{abstract}
Obesity is becoming a critical problem in the developed world and is associated with an increased incidence of osteoarthritis of the hip. The Oxford Hip Score was used to determine if Body Mass Index (BMI) is an independent factor in determining patient outcome following primary total hip arthroplasty (THA). Using data from 353 operations we found that patients with BMI $\geq 30$ had an absolute score that was lower preoperatively and postoperatively compared to those with a BMI $<30$. There was no difference in pre- and postoperative point score change within each group; Kendall's rank correlation was 0.00047 (95\% CI, -0.073 to 0.074 $(p=0.99))$ and demonstrated no trend. There was no statistically significant difference in change between those with BMI $\geq 30$ and $<30(p=0.65)$. We suggest that those with a higher BMI be considered for THA as they can expect the same degree of improvement as those with a lower BMI. Given the on-going increase in obesity these findings could be significant for the future of THA.
\end{abstract}

\section{Introduction}

Obesity is becoming a critical problem for healthcare providers in developed nations. In England the proportion of obese adults (BMI $\geq 30$ ) increased between 1993 and 2010 from $13.2 \%$ in 1993 to $26.2 \%$ in 2010 in men and from $16.4 \%$ in 1993 to $26.1 \%$ in 2010 in women [1]. Based on existing trends the prevalence of obesity is predicted to increase to $32.1 \%$ in men and $31.0 \%$ in women by the end of 2012 [2]. Body Mass Index (BMI) is commonly used to estimate body fat using an individual's weight and height [3]. The World Health Organisation defines obesity as a BMI $\geq 30[4]$.

The current trend to perform arthroplasty in younger patients who may be overweight poses the question of whether any benefit is to be gained in operating on patients with a BMI $\geq 30$.

Cooper et al. and Oliveria et al. identified the association between increasing BMI and increasing incidence of osteoarthritis of the hip $[5,6]$. This cohort of patients is, therefore, more likely to require treatment to manage their osteoarthritis, which includes surgical intervention.

The Oxford Hip Score (OHS12) is twelve-question functional outcome score [7]. It is utilised not only for research but also to determine pre- and post-op scores to ascertain improvement in individual patients with zero being the worst outcome and 48 being the best. It has been ratified in previous independent studies and can be considered to be relevant in defining patient satisfaction in elective procedures [8-10]. It is designed to be joint specific minimising the effect of comorbidity [7].

The aim of this study was to observe any difference in Oxford Hip Score between patients undergoing THA with $\mathrm{BMI} \geq 30$ and $<30$ and determine the change in Oxford Hip Score provided by surgery within each group.

\section{Method}

Between 2012 and 2014, 220 consecutive patients who had undergone THA with a BMI $\geq 30$ were identified from Bluespier orthopaedic management software. Over the same time period, 220 consecutive THAs performed in patients with $\mathrm{BMI}<30$ were identified using the same system. All 440 THAs were performed in the same centre by four consultant surgeons. All were performed via the posterior approach. All implants used were manufactured by Zimmer. Postoperative management was in line with standard departmental protocol. All patients received VTE prophylaxis. Data was missing for 87 of the selected patients. As a result, 176 THAs 
TABle 1: Preoperative Oxford Hip Scores.

\begin{tabular}{lccc}
\hline Group & Range & Standard deviation & Median \\
\hline All & $0-39$ & 6.8 & 14 \\
BMI $<30$ & $0-39$ & 6.8 & 15 \\
BMI $\geq 30$ & $0-33$ & 6.7 & 12 \\
\hline
\end{tabular}

(176 patients) were analysed in the group with a BMI $\geq 30$ and 177 THAs (177 patients) were analysed in the group with a $\mathrm{BMI}<30$.

Patient demographics including age, BMI, and ASA grade were collected [11]. Pre- and postoperative Oxford Hip Scores were collected prospectively and data was analysed retrospectively. Preoperative hips scores were recorded in the preoperative assessment clinic up to six weeks prior to surgery. Postoperative hip scores were taken at one year. A change in hip score from pre- and postoperative scores was calculated. All patients underwent elective primary THA. Patients were excluded if they had bilateral hip replacements. All patients that had previously undergone lower limb joint arthroplasty were excluded from the study. All cases were for primary hip osteoarthritis.

The ASA grade was assigned before surgery and relates to the systemic condition of a patient. It is graded from one to five, with one indicating a fit patient, two indicating mild systemic disease, three indicating severe systemic disease, four indicating imminent risk of death due to illness, and five indicating a moribund patient with death as the expected outcome.

Descriptive and statistical analysis was performed using StatsDirect (StatsDirect Ltd., 2013). Mann-Whitney $U$ tests were performed to determine if there was a difference between the two BMI groups with regard to age difference, before and after Oxford Hip Scores and ASA grade. Kendall's rank correlation was calculated for BMI and ASA grade. Statistical significance was set at $p<0.05$.

\section{Result}

The median age in the BMI $<30$ was 68 (range 56-60) and $\mathrm{BMI} \geq 30$ was 66 (range $52-74$ ). There was no statistical difference between the two groups $(p=0.35)$.

There were 90 males and 86 females in the $<30$ BMI group. There were 85 males and 92 females in the $\geq 30 \mathrm{BMI}$ age group. There was no statistically significant difference in ages between the two BMI groups $(p=0.55)$.

3.1. Preoperative Oxford Scores. The median scores were 14, 15 , and 12 for all BMI $<30$ and BMI $\geq 30$ groups, respectively (Table 1). There was a statistically significant difference between the two BMI groups and preoperative Oxford Hip Score $(p=0.0002)$. A negative correlation exists between BMI and preoperative Oxford Hip Score; Kendall's rank correlation is -0.15 (CI 95\%: -0.22 to $-0.80(p \leq 0.0001)$ ). This was statistically significant, indicating that an increase in BMI is associated with a worse preoperative Oxford Hip Score (Figure 1).

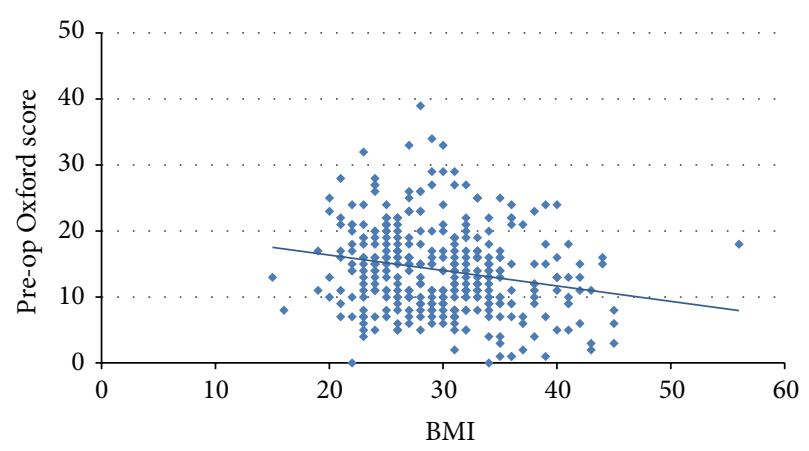

FIGURE 1: BMI versus pre-op Oxford score.

TABLE 2: Postoperative Oxford Hip Scores.

\begin{tabular}{lccc}
\hline Group & Range & Standard deviation & Median \\
\hline All & $8-48$ & 7.1 & 43 \\
BMI $<30$ & $11-48$ & 4.74 & 44.5 \\
BMI $\geq 30$ & $8-48$ & 7.29 & 42 \\
\hline
\end{tabular}

TABle 3: Change in Oxford Hip Scores.

\begin{tabular}{lccc}
\hline Group & Range & Standard deviation & Median \\
\hline All & -8 to 47 & 8.1 & 28 \\
BMI $<30$ & -8 to 42 & 6.43 & 28 \\
BMI $\geq 30$ & -7 to 47 & 8.35 & 28 \\
\hline
\end{tabular}

TABLE 4: ASA grade.

\begin{tabular}{lccc}
\hline Group & Range & Standard deviation & Median \\
\hline All & 1 to 4 & 0.47 & 2 \\
BMI $<30$ & 1 to 4 & 0.40 & 2 \\
BMI $\geq 30$ & 1 to 4 & 0.49 & 2 \\
\hline
\end{tabular}

3.2. Postoperative Oxford Scores. The median scores were $43,44.5$, and 42 for all $\mathrm{BMI}<30$ and $\mathrm{BMI} \geq 30$ groups, respectively (Table 2 ). There was a statistically significant difference between the two BMI groups and postoperative Oxford Hip Score $(p=0.0001)$. There was statistically significant negative correlation between BMI and postoperative Oxford Hip Score; Kendall's rank correlation is -0.13 (95\% CI, -0.19 to $-0.06(p=0.0008))$. This indicates that an increase in $\mathrm{BMI}$ is associated with a worse absolute postoperative Oxford Hip Score (Figure 2).

3.3. Change in Oxford Hip Scores. The median change for each group was the same at 28 points (Table 3 ). There was no statistical difference between the two BMI groups $(p=0.65)$. There was no correlation between BMI and change in Oxford Hip Score; Kendall's rank correlation is 0.00047 (95\% CI, -0.073 to $0.074(p=0.9902))$. This demonstrates that there is no relationship between BMI and change in Oxford Hip Score (Figure 3).

3.4. ASA Grade. The median ASA scores were 2 for all groups, respectively (Table 4 ). There was no statistically significant 


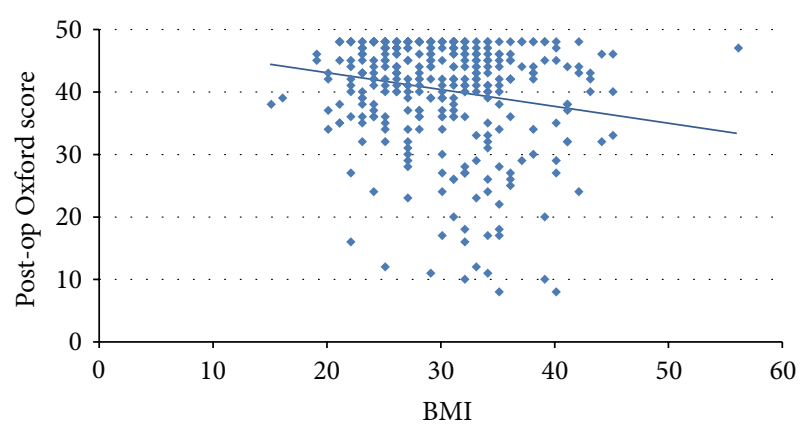

FiguRE 2: BMI versus post-op Oxford score.

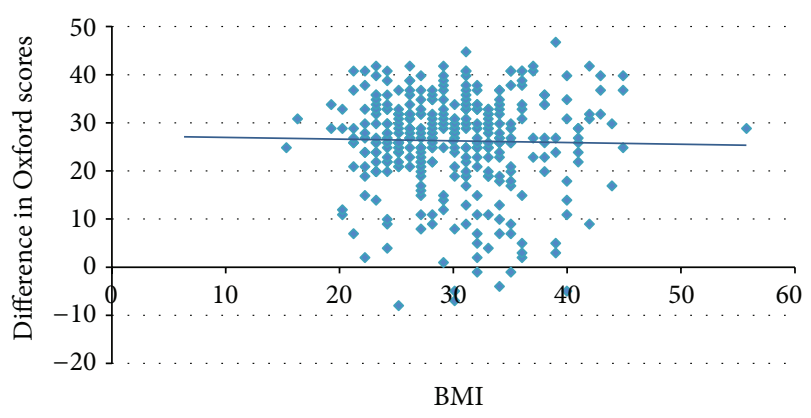

FIGURE 3: BMI versus difference in Oxford scores.

difference between the ASA grade and the two BMI groups $(p=0.1)$. There was a statistically significant correlation between increasing BMI and increasing ASA grade (higher ASA grade indicates worse systemic condition); Kendall's rank correlation is 0.13 (95\% CI: 0.065 to $0.19(p=0.007)$ ) (Figure 4).

Seven cases (1.98\%) had a worse OHS12 postoperatively than preoperatively. Of these, 6 were in the BMI $\geq 30$ group and only one was in the BMI $<30$ group. In this subgroup of patients there was no statistically significant correlation between BMI and change in Oxford Hip Score.

\section{Discussion}

This paper demonstrates that patients in a higher BMI category have a significantly lower absolute Oxford Hip Score both before and after surgery. Patients with a BMI $\geq 30$ experience the same change in Oxford Hip Score as patients with a BMI $<30$ (median 28-point difference).

Judge et al. have analysed patient satisfaction with Oxford Hip Score and concluded a $97.6 \%$ satisfaction rate with a change of 14 points compared to $81.8 \%$ with a change below this threshold. At 6 months they found an absolute score of $>35$ associated with the highest satisfaction. They also noted that patients with a worse pre-op score still gained the highest satisfaction with a lower post-op score as long as the difference in change was 14 points or more [12]. Our paper did not look specifically at patient satisfaction; however, the improvement in score would suggest, based on current literature, greater satisfaction in our cohort with both groups scoring $>35$ at 12 months and $>14$ points increase post-op.

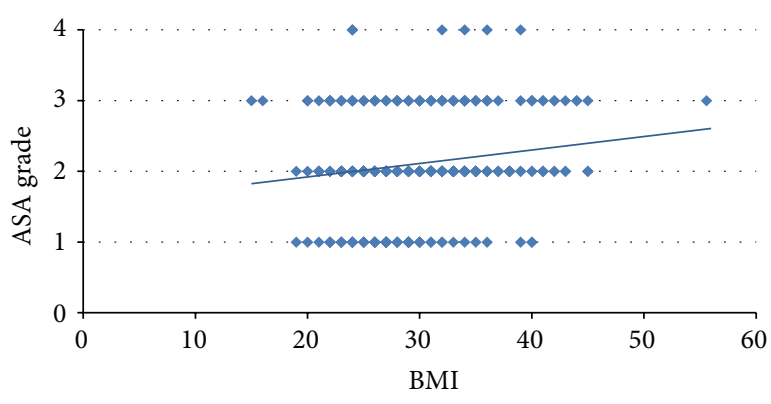

Figure 4: BMI versus ASA grade.

Existing evidence suggests that BMI should not be a discriminatory factor in patient selection for THA. Michalka et al. found that patients with a $\mathrm{BMI} \geq 30$ gain similar benefit from THA as patients with a BMI $<30$ [13]. Andrew et al. assessed the influence of BMI on change in Oxford Hip Score pre- and postoperatively. They concluded that there was no difference between those patients in different BMI categories, suggesting that obese and morbidly obese patients have the same clinical benefit as nonobese patients. They do acknowledge increasing comorbidity such as ischaemic heart disease, diabetes, apnoeic sleep disorders, and hypertension in the obese category, all of which could increase the risk of anaesthesia. However, they conclude the perceived belief that patients with higher BMIs do not clinically benefit following surgery is a misconception. Higher BMIs should not deter the surgeon from performing a THR [14].

This study has several limitations. First, potential bias may exist as some surgeons were more willing to perform THA surgery on those with a higher BMI compared to their colleagues; however, with a relatively equal number of patients in each group we do not suspect bias at the primary care referral level. Second, the type of prosthesis used for each patient was not recorded; therefore, any potential difference in outcome which could be prosthesis-dependent is not known. However, all implants were Orthopaedic Data Evaluation Panel (ODEP) rated and approved for implant and have demonstrated performance within guidelines. Third, complication rates were not assessed in this study. Other papers have reported conflicting outcomes on this. Lübbeke et al. compared the incidence of complications in obese and nonobese patients undergoing primary THA. They found that obesity substantially increased the infection rate in women but not in men and that the incidence of dislocation was higher in obese women than in nonobese women [15]. Andrew et al. identified no difference in complication rate (dislocation, revision rates, increased haemorrhage, deep infection, deep-venous thrombosis, and pulmonary embolism) or radiological change at five years between obese and nonobese patients [14]. We would suggest that an RCT or a prospective cohort study comparing these two groups would go someway in reducing confounding bias and the limitations this study has.

Our study indicates that THA in patients with BMI > 30 does not lead to a reduction in improvement in Oxford Hip Score compared to those with BMI $<30$. Patients with 
a BMI $\geq 30$ showed the same increase in Oxford score as those with a BMI < 30 but had lower absolute Oxford Hip Scores both before and after surgery.

We, therefore, recommend that patients with a higher BMI be considered candidates for THA, as they can expect the same degree of change in improvement on Oxford Hip Score. The caveat is that absolute score should not be expected to equal that of patients with a lower BMI.

\section{Conflict of Interests}

The authors declare that there is no conflict of interests regarding the publication of this paper.

\section{References}

[1] The NHS Information Centre, Lifestyles Statistics, Paul Eastwood, Lifestyle Statistics Section Head, 2012, http://www.ic.nhs.uk.

[2] P. Zaninotto, J. Head, E. Stamatakis, H. Wardle, and J. Mindell, "Trends in obesity among adults in England from 1993 to 2004 by age and social class and projections of prevalence to 2012," Journal of Epidemiology and Community Health, vol. 63, no. 2, pp. 140-146, 2009.

[3] V. S. Hubbard, "Defining overweight and obesity: what are the issues?" The American Journal of Clinical Nutrition, vol. 72, no. 5, pp. 1067-1068, 2000.

[4] N. K. Arden, A. Kiran, A. Judge et al., "What is a good patient reported outcome after total hip replacement?" Osteoarthritis and Cartilage, vol. 19, no. 2, pp. 155-162, 2011.

[5] C. Cooper, H. Inskip, P. Croft et al., "Individual risk factors for hip osteoarthritis: obesity, hip injury and physical activity," American Journal of Epidemiology, vol. 147, no. 6, pp. 516-522, 1998.

[6] S. A. Oliveria, D. T. Felson, P. A. Cirillo, J. I. Reed, and A. M. Walker, "Body weight, body mass index, and incident symptomatic osteoarthritis of the hand, hip, and knee," Epidemiology, vol. 10, no. 2, pp. 161-166, 1999.

[7] D. W. Murray, R. Fitzpatrick, K. Rogers et al., "The use of the Oxford hip and knee scores," The Journal of Bone and Joint Surgery-British Volume, vol. 89, no. 8, pp. 1010-1014, 2007.

[8] J. Dawson, R. Fitzpatrick, A. Carr, and D. Murray, "Questionnaire on the perceptions of patients about total hip replacement," The Journal of Bone \& Joint Surgery Series B, vol. 78, no. 2, pp. 185-190, 1996.

[9] Y. Kalairajah, K. Azurza, C. Hulme, S. Molloy, and K. J. Drabu, "Health outcome measures in the evaluation of total hip arthroplasties-a comparison between the harris hip score and the Oxford hip score," Journal of Arthroplasty, vol. 20, no. 8, pp. 1037-1041, 2005.

[10] M. Ostendorf, H. F. van Stel, E. Buskens et al., "Patientreported outcome in total hip replacement. A comparison of five instruments of health status," The Journal of Bone \& Joint Surgery Series B, vol. 86, no. 6, pp. 801-808, 2004.

[11] R. D. Dripps, "New classification of physical status," Anaesthesiology, vol. 24, article 111, 1963.

[12] A. Judge, N. K. Arden, A. Kiran et al., "Interpretation of patientreported outcomes for hip and knee replacement surgery: identification of thresholds associated with satisfaction with surgery," The Journal of Bone \& Joint Surgery-British Volume, vol. 94, no. 3, pp. 412-418, 2012.
[13] P. K. R. Michalka, R. J. K. Khan, M. C. Scaddan, S. Haebich, N. Chirodian, and J. A. Wimhurst, "The influence of obesity on early outcomes in primary hip arthroplasty," Journal of Arthroplasty, vol. 27, no. 3, pp. 391-396, 2012.

[14] J. G. Andrew, J. Palan, H. V. Kurup, P. Gibson, D. W. Murray, and D. J. Beard, "Obesity in total hip replacement," The Journal of Bone and Joint Surgery-British Volume, vol. 90, no. 4, pp. 424-429, 2008.

[15] A. Lübbeke, R. Stern, G. Garavaglia, L. Zurcher, and P. Hoffmeyer, "Differences in outcomes of obese women and men undergoing primary total hip arthroplasty," Arthritis \& rheumatism, vol. 57, no. 2, pp. 327-334, 2007. 


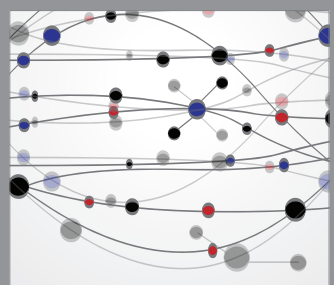

The Scientific World Journal
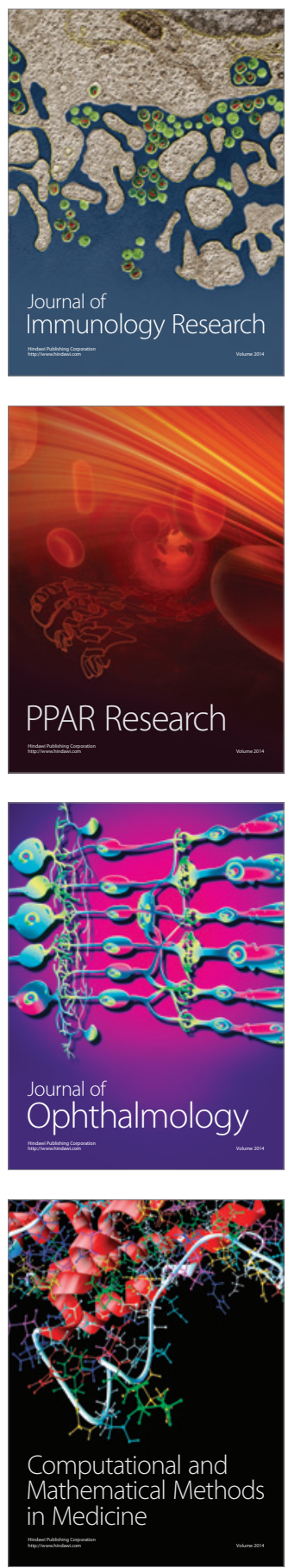

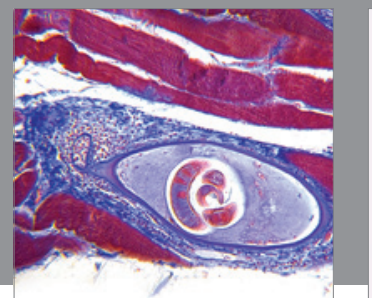

Gastroenterology

Research and Practice
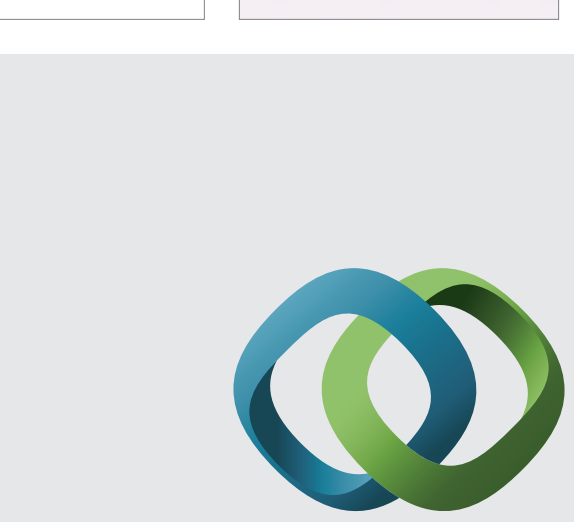

\section{Hindawi}

Submit your manuscripts at

http://www.hindawi.com
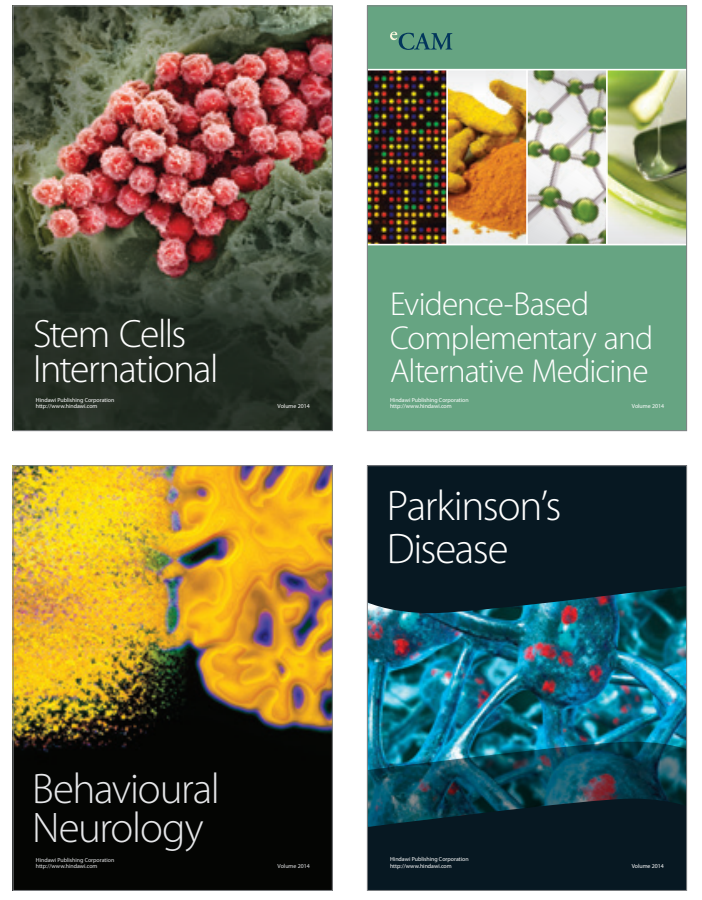
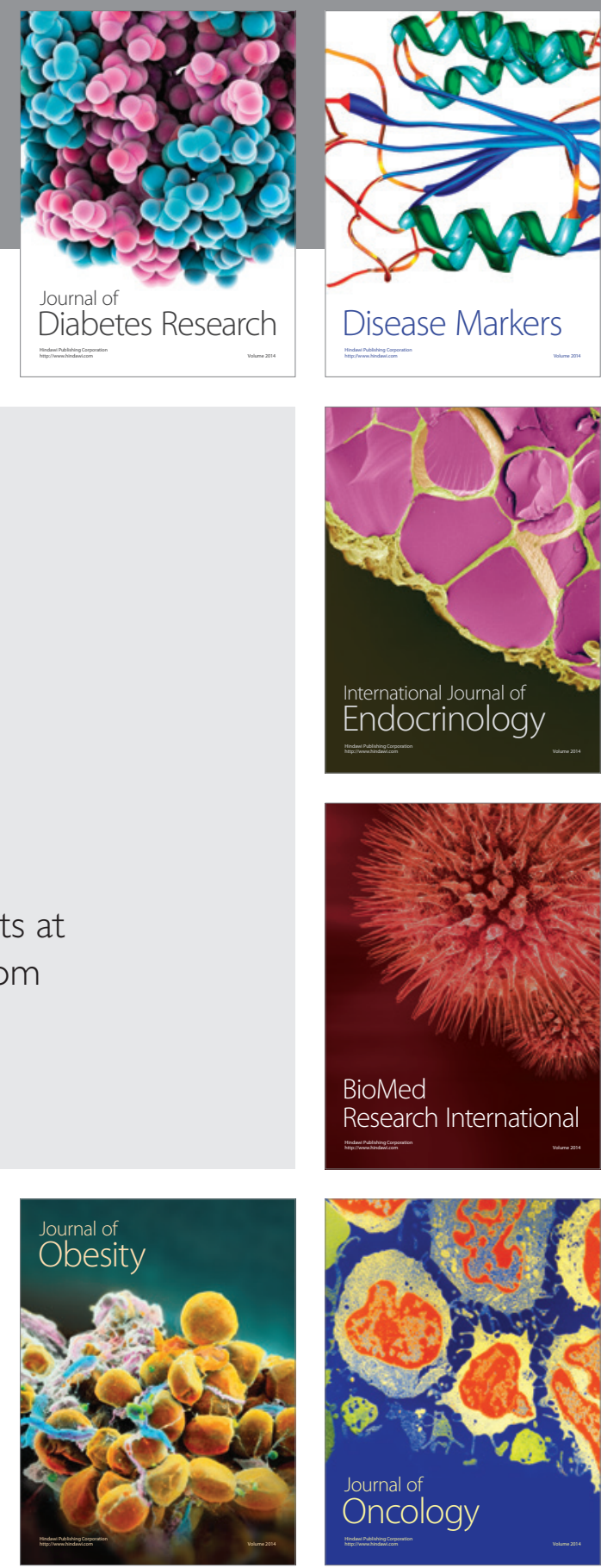

Disease Markers
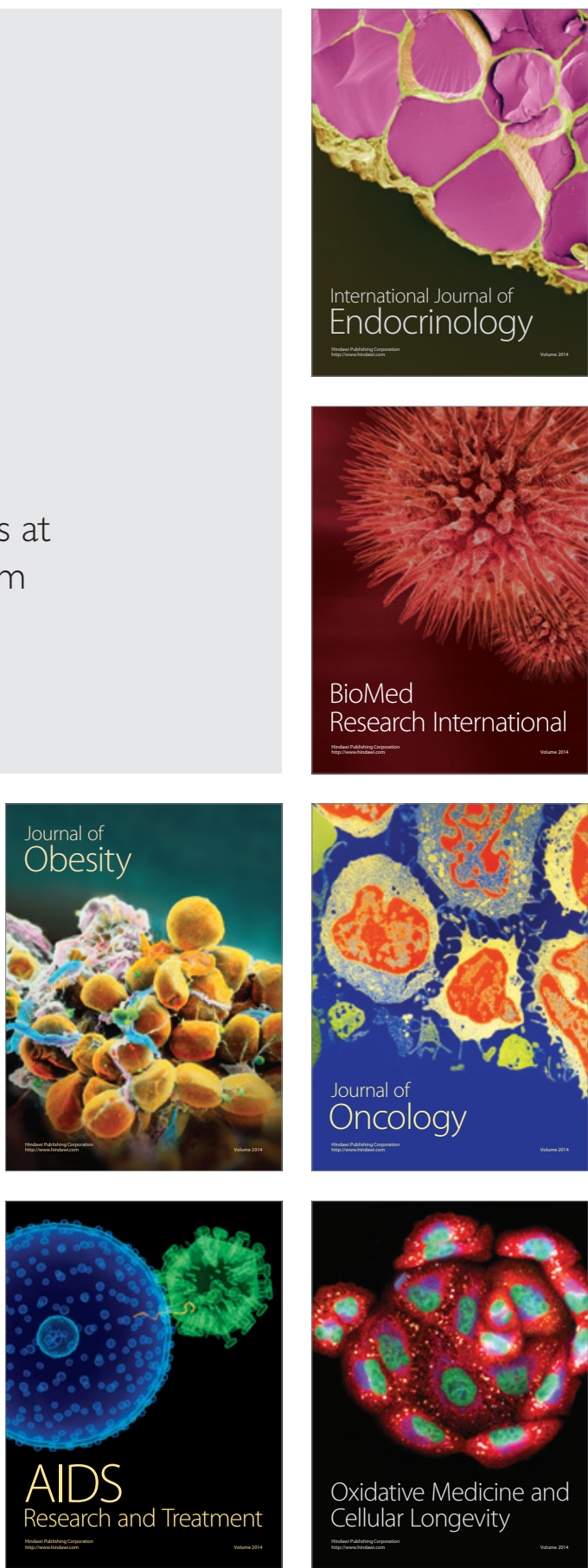